\title{
Segregation-Induced Nanofaceting Transition at an Asymmetric Tilt Grain Boundary in Copper
}

\author{
Nicolas J. Peter, ${ }^{1, *}$ Timofey Frolov, ${ }^{2}$ Maria J. Duarte, ${ }^{1}$ Raheleh Hadian, ${ }^{1}$ Colin Ophus, ${ }^{3}$ Christoph Kirchlechner, ${ }^{1}$ \\ Christian H. Liebscher, ${ }^{1, *}$ and Gerhard Dehm ${ }^{1}$ \\ ${ }^{1}$ Max-Planck Institut für Eisenforschung GmbH, 40237 Düsseldorf, Germany \\ ${ }^{2}$ Lawrence Livermore National Laboratory, Livermore, California 94550-9234, USA \\ ${ }^{3}$ National Center for Electron Microscopy, Molecular Foundry, Lawrence Berkeley National Laboratory, \\ Berkeley, California 94720, USA
}

(Received 28 May 2018; revised manuscript received 23 October 2018; published 20 December 2018)

\begin{abstract}
We show that chemistry can be used to trigger a nanofaceting transition. In particular, the segregation of Ag to an asymmetric tilt grain boundary in $\mathrm{Cu}$ is investigated. Aberration-corrected electron microscopy reveals that annealing the bicrystal results in the formation of nanometer-sized facets composed of preferentially Ag-segregated symmetric $\Sigma 5\{210\}$ segments and Ag-depleted $\{230\} /\{100\}$ asymmetric segments. Our observations oppose an anticipated trend to form coarse facets. Atomistic simulations confirm the nanofacet formation observed in the experiment and demonstrate a concurrent grain boundary phase transition induced by the anisotropic segregation of $\mathrm{Ag}$.
\end{abstract}

DOI: 10.1103/PhysRevLett.121.255502

Grain boundaries (GBs), as internal interfaces in polycrystals, strongly influence many properties of structural and functional materials [1]. Changes in grain boundary structure due to temperature, pressure, or chemistry can have a pronounced effect on GB energy, mobility, and cohesive strength ultimately influencing the macroscopic properties of these materials [2]. For example, Duscher et al. observed a monolayer of $\mathrm{Bi}$ atoms segregating to a $\Sigma 5$ $\mathrm{GB}$ in $\mathrm{Cu}$, and it was proposed that bonding effects or the atomic size mismatch are causing embrittlement of otherwise ductile $\mathrm{Cu}[3,4]$. Recent experimental studies suggested that abrupt changes in materials properties including diffusivity [5] and grain growth [6] can be explained by structural transitions at GBs [2]. While these studies linked the discontinuous changes in GB structure to important materials properties such as abnormal grain growth in ceramics [6], liquid metal embrittlement [7], and activated sintering [8], the atomic structure of these GB phases remains elusive. Theoretical investigation of transitions at interfaces dates back to some work of Gibbs, who considered the possibility of different interfacial states and derived the criteria for their equilibrium and stability [9]. Transitions at planar GBs were later discussed by Hart [10], who referred to them as "two-dimensional phase transformations." More recently, transitions at planar GBs were

Published by the American Physical Society under the terms of the Creative Commons Attribution 4.0 International license. Further distribution of this work must maintain attribution to the author(s) and the published article's title, journal citation, and DOI. studied with phase field models [11], atomistic simulations [12], and lattice-gas models [13].

In addition to the conventional thermodynamic variables that could trigger changes in GB structure, Cahn introduced orientational degrees of freedom and categorized transitions at GBs into congruent and faceting types [14]. He suggested that faceting transitions should be much more common than the congruent transitions at planar boundaries. Temperatureinduced GB faceting was reported in experimental studies [15-17] and facet formation mechanisms were explored by atomistic simulations in single component systems [18-22]. On the other hand, the effect of solute segregation on faceting is much less understood. The evolution of microscopically faceted boundaries was observed in $\mathrm{Bi}$ doped $\mathrm{Cu}$ bicrystals with increasing temperature and Bi concentration [23]. The direct observation of a reversible faceting transition in a welldefined boundary was demonstrated only in a single study by Ference and Balluffi for the $\mathrm{Cu}-\mathrm{Bi}$ system [24]. However, both studies did not resolve the atomic structure of the segregated boundary and detected only relatively large facets with the size of several tens of nanometers and larger. More recently, Kundu et al. established a bilayer Bi segregation at about $100 \mathrm{~nm}$ long facets in a faceted $\mathrm{Cu} \mathrm{GB}$, but $\mathrm{Bi}$ decoration of the other facet type could not be determined [25]. On the other hand, stable nanofaceted GBs were observed using high resolution scanning transmission electron microscopy (STEM) in elemental systems and could potentially exist in doped systems [26,27]. Such small facets can be observed only with atomic scale resolution and could have been overlooked by previous studies.

In this Letter we use atomic resolution STEM, atom probe tomography (APT), and atomistic simulations to 
demonstrate a nanoscale faceting transition in an asymmetric $\mathrm{Cu}$ tilt grain boundary induced by segregation of $\mathrm{Ag}$. The choice of the material system is motivated by recent measurements of $\mathrm{Ag}$ diffusion [5] in a symmetric $\Sigma 5 \mathrm{Cu}$ GB that reported a non-Arrhenius diffusion behavior and atomistic simulations that demonstrated a first-order transition in the same symmetric boundary [28].

$\mathrm{A} \mathrm{Cu}$ bicrystal was grown by the Bridgman technique. The [001] axes of both seed crystals were parallel to the growth direction. The grains were misoriented by $54^{\circ}$, forming an asymmetric, noncoinciding lattice tilt boundary with a $31^{\circ}$ inclination angle. To unambiguously prove a chemical trigger for a GB transition, ideally in situ segregation at atomic resolution would need to be performed at high temperatures. Since this is even now hard to realize in situ, the following approach was selected. The structure of the asymmetric GB was investigated following three different treatments with three different samples: (1) the pure asgrown state, (2) the pure $\mathrm{Cu}$ reference state annealed at $800^{\circ} \mathrm{C}(1073 \mathrm{~K})$ for $120 \mathrm{~h}$ under high vacuum conditions, and (3) the Ag-segregated state after an annealing of sputter coated samples under the same conditions as in (2). All experimental details can be found in the Supplemental Material [29]. Representative high-angle annular dark-field (HAADF) STEM micrographs of each state of the GB are given in Figs. 1(a)-1(c). The GB plane determined using the stereographic projection was close to the orientation $\{110\} /\{410\}$, where the indices correspond to the crystallographic planes of the abutting grains parallel to the GB plane [Fig. 1(a)]. The overall $\mathrm{Cu}$ boundary appears to be macroscopically flat, with a certain roughness or short facets being visible at the atomic scale. The planar shape of the boundary suggests a weak inclination dependence of the GB energy (flat Wulff shape), which is consistent with published data

(a) As grown

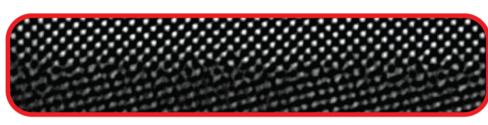

(c) Annealed w/ Ag

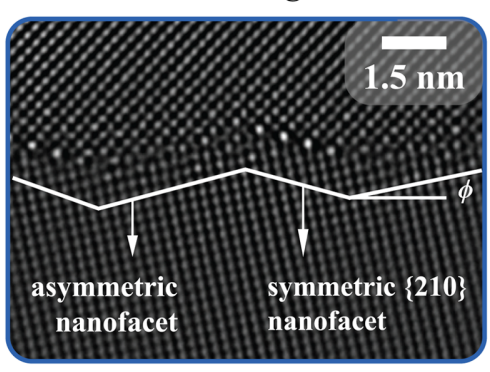

(b) Annealed w/o Ag
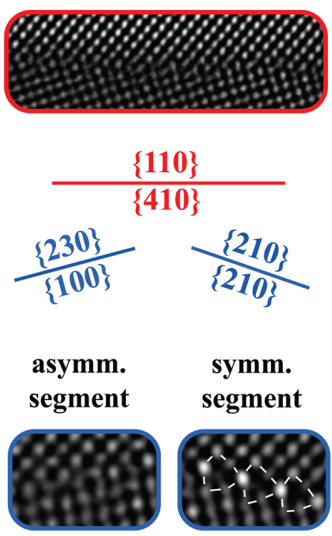

FIG. 1. HAADF STEM micrographs in [001] zone axis orientation of (a) the relatively flat as-grown GB structure, (b) the relatively flat annealed GB structure, and (c) the Ag-segregated GB structure exhibiting distinct faceting with preferential segregation to the symmetric $\{210\}$ segments. The scale is applicable to all three micrographs. obtained using atomistic calculations at $0 \mathrm{~K}$ [18]. After annealing, the pure $\mathrm{Cu}$ GB remained flat, and its inclination did not change significantly [Fig. 1(b)]. The new GB plane orientation was close to $\{110\} /\{310\}$, which is only a few degrees away from the as-grown state, confirming the inclinational isotropy of the GB energy in pure $\mathrm{Cu}$.

Very different behavior was observed in the sample doped with Ag. After annealing, the GB distinctly faceted at the atomic scale into an asymmetric segment close to $\{230\} /$ $\{100\}$, and a symmetric $\Sigma 5\{210\}$ segment [Fig. 1(c)]. Based on a section of the boundary with about 30 facets, segment lengths were measured to be $1.9 \pm 0.9 \mathrm{~nm}$ and $1.8 \pm 0.5 \mathrm{~nm}$ for the asymmetric and symmetric parts, respectively. The angle $\phi$ between the horizontal average boundary plane in the reference samples and the two facet segments is $\sim 15^{\circ}$, as indicated in Fig. 1(c). Bright atomic columns at the symmetric facet segments indicate Ag containing columns with a higher $Z$ number than the surrounding grain interiors [35]. Consequently, preferential segregation to the symmetric segments was observed, while asymmetric segments contained little to no $\mathrm{Ag}$ atoms. In the symmetric facet segment $\mathrm{Ag}$ atoms occupy from $50 \%$ to $100 \%$ of the atomic columns located at the tips of the kite-shaped structural units, and we find evidence of beam-induced GB diffusion with the $\mathrm{Ag}$ atoms moving between different lattice sites [36].

The volume reconstruction of a boundary containing an APT tip clearly identifies Ag solute excess along the GB plane with a peaking line profile of up to about 2 at. $\%$, while the grain interior contains 0.16 at. $\% \mathrm{Ag}$ - averaged from six different samples [Fig. 2(b)]. The APT measurements
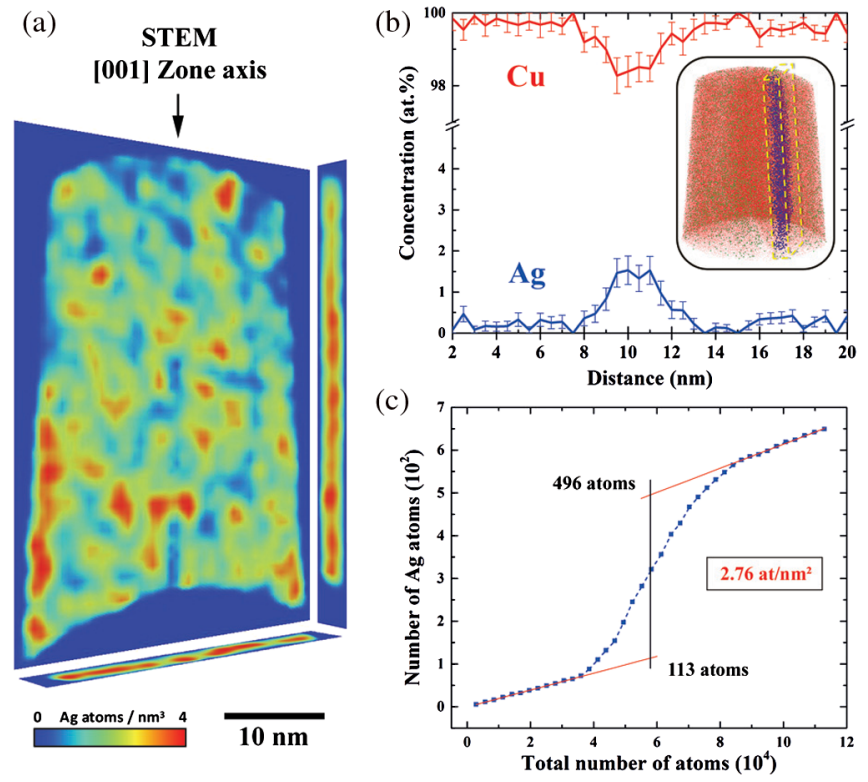

FIG. 2. 3D APT reveals the inhomogeneous segregation of Ag to the asymmetric GB. (a) The projected Ag density plot of the GB. (b) 1D line profile across the boundary. (Inset) The APT reconstructed volume. (c) The Ag solute excess atom concentration determined across the GB. 
provide evidence that the GB is locally enriched in $\mathrm{Ag}$, in agreement with the HAADF STEM results [Fig. 1(c)]. The 2D Ag density map reveals a nonhomogeneous decoration of the GB plane along the tilt axis (not accessible by STEM), in agreement with the observed preferential segregation and implying an in-depth reconstruction of the GB [Fig. 2(a)]. Such reconstructions were previously demonstrated by simulations and could be due to thermal fluctuations [37]. Another possibility is that the GB exhibits in-depth faceting-defaceting transitions induced by segregation [38]. The combination of STEM and APT establishes a correlation between $\mathrm{Ag}$ enriched areas and different structural motifs of the GB. The Ag content at the GB was quantified by calculating the atomic interfacial excess following Refs. [39,40] [Fig. 2(c)]. A solute excess concentration of 2.76 at. $/ \mathrm{nm}^{2}$ was determined as an average value for the whole boundary.

To get further insights into the atomic details of the segregation induced faceting transition found in the experiment, we performed atomistic simulations. Here we used the combined Monte Carlo and molecular dynamics (MCMD) approach implemented in LAMMPS [41]. The GB (image) simulation methodology is described in the Supplemental Material [29] and in previous work [12,42,43]. Figure 3

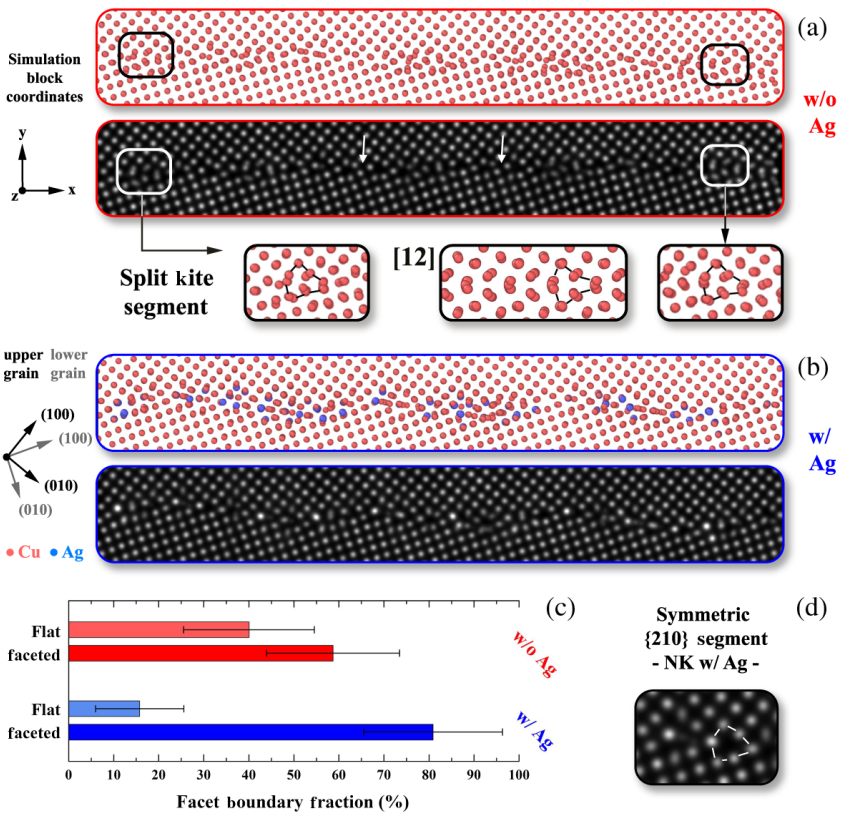

FIG. 3. MCMD simulation results and the corresponding STEM image simulations for the annealed asymmetric $\mathrm{Cu}$ GB (a) without $\mathrm{Ag}$. The highlighted areas are enlarged below the image simulation on the left and right sides to show the rare presence of split kite structures. As a split kite reference, the central structure is adopted with permission from Ref. [12]. Arrows indicate the location of GB disconnections. The MCMD simulation results with (b) the presence of $\mathrm{Ag}$ and (c) the respective statistical analysis results. An enlarged area of a symmetric $\{210\}$ facet segment containing $\mathrm{Ag}$ is presented with the normal kite structure highlighted in (d). shows two representative GB structures with Ag (blue boxes) and without Ag (red boxes). Using these structures, STEM images were simulated using PRISMATIC code [34]. The structure of the $\mathrm{GB}$ in pure $\mathrm{Cu}$ at $600 \mathrm{~K}$ is shown in Fig. 3(a) to be relatively flat. Examination of different snapshots reveals small structural units of symmetric boundaries that appear as a result of thermal fluctuations. Specifically, the $\mathrm{Cu}$ embedded atom potential predicts three different GB phases for the $\Sigma 5\{210\}$ symmetric boundary which were identified in Ref. [12] and called split kite (SK), filled kite (FK), and normal kite (NK). Small units of SK and FK can be identified in the image. In the $\mathrm{Cu}-\mathrm{Ag}$ system the boundary structure is noticeably different. In agreement with our experimental findings, the GB faceted into symmetric $\{210\}$ and asymmetric $\{230\} /\{100\}$ segments, with large $\mathrm{Ag}$ atoms preferentially segregating to the $\{210\}$ segments. The asymmetric and symmetric facet lengths were found to be $1.4 \pm 0.4$ and $2.1 \pm 0.7 \mathrm{~nm}$, respectively, and the facet inclination angle $\phi$ was close to $15^{\circ}$ on average, close to the experimental findings above. The preferential segregation of $\mathrm{Ag}$ atoms to symmetric segments is even more apparent in the simulated STEM images that show portions of the boundary with a higher intensity of $\mathrm{Ag}$ [compare to the enlarged area in Fig. 3(d)]. Moreover, in the faceted boundary we no longer observe the SK structural units found in pure $\mathrm{Cu}$. This further confirms that a structural transformation took place and is consistent with a similar destabilization of the SK structure by Ag that has been previously demonstrated for a symmetric $\Sigma 5\{210\}$ boundary [28,42]. To quantify the structural transformation observed in the simulations, we analyzed the size of the fluctuating facets in both the $\mathrm{Cu}$ and the $\mathrm{Cu}-\mathrm{Ag}$ system. At $600 \mathrm{~K}$ in pure $\mathrm{Cu}$, the ratio of flat to faceted boundary parts reaches almost unity, while in the case of the Ag-segregated boundary, the nanofacet fraction increased up to $80 \%$ on average. Thus, our experimental results and simulations are in qualitative agreement with each other in terms of the segregation-induced faceting, although the exact structures of the symmetric segments show some variation. It is important to note that the experimental structure was observed at room temperature, while simulations are performed at high temperature due to timescale limitations of the modeling.

To make a better connection with room temperature structures found in the experiments, we performed GB segregation and free energy calculations for the $\{210\}$ symmetric GB at $300 \mathrm{~K}$. Specifically, we investigate the possibility of a segregation-induced transition to the normal kite structure, which could take place at lower temperatures. Similar transitions have been demonstrated at high temperature in our previous studies $[28,42]$, but also recently with a symmetry breaking structural transition in the Ni-Mo system [44]. These calculations would also imply a possibility of a hierarchical phase transformation path, which could be difficult to observe experimentally. Using the MCMD approach with periodic boundary conditions, we modeled segregation to the three different GB 
phases of the symmetric $\Sigma 5\{210\}[001] \mathrm{GB}$ at $300 \mathrm{~K}$ $[28,42]$. The simulations were performed at six different values of the chemical potential difference $\Delta \mu=\mu_{\mathrm{Ag}}-\mu_{\mathrm{Cu}}$ to sample different concentrations of Ag. The snapshots of the boundary plane edge-on represent equilibrium segregation at a chemical potential of $0.55 \mathrm{eV}$, the same $\Delta \mu$ value used for faceting simulations. The snapshots reveal that while the SK phase contains only a few Ag atoms, the NK phase accommodates almost a monolayer of $\mathrm{Ag}$ for the same value of $\Delta \mu$ or concentration of $\mathrm{Ag}$ in the bulk. Figure 4(a) shows the $\left[N_{\mathrm{Ag}}\right]_{N}$ plots as a function of $\Delta \mu$ which quantify this strong effect of GB structure on impurity segregation. We calculated the reduction in GB free energy of each of the three phases due to $\mathrm{Ag}$ by integrating the adsorption equation as $\gamma_{0}-\gamma=-\int\left[N_{\mathrm{Ag}}\right]_{N} d \Delta \mu$ [9] [Fig. 4(b)]. The higher uptake of $\mathrm{Ag}$ by the NK structure effectively lowers the boundary free energy by a maximum value of $5 \mathrm{~mJ} / \mathrm{m}^{2}$, making it the most thermodynamically favorable configuration at higher $\mathrm{Ag}$ concentrations. The free energy analysis demonstrates that at room temperature the symmetric segments of the faceted boundary should have NK structure, which was also observed in the experiment. Thus, our high temperature simulations and the free energy analysis combined are in agreement with the experimentally observed nanofaceted structure. The same simulations were

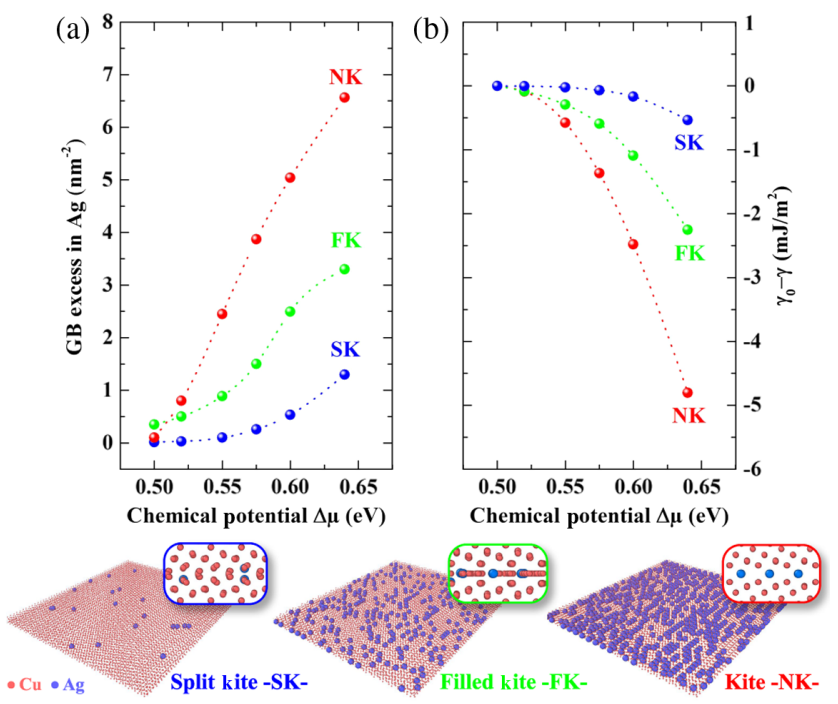

FIG. 4. MCMD calculations of the equilibrium Ag segregation and GB free energy for the three possible kite structures of the symmetric tilt $\Sigma 5\{210\}[001]$ boundary at $300 \mathrm{~K}$. (a) The equilibrium segregation as a function of the chemical potential difference. Ag segregation for the NK can be almost an order of magnitude higher than for the SK structure. (b) The reduction in the GB free energy as a function of chemical potential difference indicates that Ag stabilizes the NK structure. (Bottom panels) GB planes of each structure with equilibrium segregation of $\mathrm{Ag}$ for the same value of the chemical potential difference $\Delta \mu=0.55 \mathrm{eV}$ (same bulk composition). In the NK structure Ag atoms form almost a monolayer at the boundary, while the denser [12] SK structure has only a few Ag atoms. performed for the asymmetric facet segment as well, supporting the above findings. These results can be found in the Supplemental Material [29].

The experimental observations and atomistic simulations suggest the following mechanism for the Ag-triggered faceting transition. At first, annealing the $\mathrm{Ag}$ coated $\mathrm{Cu}$ bicrystal at $800{ }^{\circ} \mathrm{C}$ completely dissolves $\mathrm{Ag}$ in $\mathrm{Cu}$ [45]. This agrees well with APT measurements, that reveal a bulk Ag concentration of 0.16 at.\%, allowing an estimation of the onset temperature of segregation to be $\sim 500{ }^{\circ} \mathrm{C}$ during slow cooling according to the equilibrium phase diagram [45]. The simulation results show a significant increase in facet fraction for the GB annealed in the presence of $\mathrm{Ag}$ at elevated temperatures and demonstrate that absorbed $\mathrm{Ag}$ atoms preferentially segregate to symmetric $\{210\}$ segments. Our study shows that the segregation to GBs can be very anisotropic and can trigger nanofaceting transitions. The factors that control the faceted shape of a GB and the size of the facets include inclinational anisotropy of the GB free energy, elastic effects due to GB stress of different facets [21], and the elastic interaction between disconnections formed by GB facet junctions [27]. Our experiments and simulations demonstrate a faceted GB composed of Ag-rich and Ag denuded segments. According to the Gibbs adsorption equation and the free energy calculations performed in this work, the uneven segregation should promote stronger inclinational anisotropy. Segregation has a strong effect on GB stress, which is another factor controlling GB faceting. GB stress calculations for a $\Sigma 5$ symmetric tilt boundary in the same model system showed that initially tensile GB stress could be reduced to zero and could even become compressive as a result of segregation of large $\mathrm{Ag}$ atoms [46]. Since segregation depends on the inclination, this strong change in GB stress is expected to be different in different GB facets and to promote faceting. Finally, the interaction between the disconnections may be affected by segregation as it changes the excess volumes of the different GB facets. All of these energetic factors are strongly coupled to segregation. Therefore, it is difficult to distill what is the most important in our case. However, it is clear that impurity segregation promotes anisotropy and faceting. Our experiments confirm the weak inclinational anisotropy of the GB free energy in pure $\mathrm{Cu}$ [18]. However, the structures we observe in both the experiment and hightemperature simulations do not match previously published structures generated at $0 \mathrm{~K}$ composed of $\{310\}$ and $\{210\}$ kite motifs.

From a kinetic perspective the Ag decorated symmetric $\{210\}$ facets start forming at $\sim 500{ }^{\circ} \mathrm{C}$, assisted by thermally induced fluctuations in facet formation and dissolution. Consequently, intermediate asymmetric $\{230\} /\{100\}$ segments form through step nucleation and migration to preserve the macroscopic GB plane orientation. According to $\mathrm{Wu}$ et al. [20], facet coarsening requires the migration of the symmetric segment perpendicular to its plane along the 
asymmetric segment until it meets another symmetric facet. Since facet coarsening is related to the atomic mismatch on both sides of a grain boundary facet, the symmetric $\{210\}$ segments are expected to exhibit a low energy barrier for coarsening, but strongly reduced coarsening is expected for the asymmetric segments. This is in good agreement with our experimental findings and implies that facet coarsening is kinetically limited, likely influencing microstructure evolution of such alloys. Two recent studies calculated the impact of solute segregation at symmetric $\Sigma 5$ GBs on macroscopic properties such as GB diffusion and sliding $[47,48]$. In light of our findings, this emphasizes that the observed segregation-induced nanofaceting transition will have a strong influence on the interfacial properties since it goes even beyond the classical picture of LangmuirMcLean-type segregation.

In conclusion, we present in this Letter the experimental observation of a chemically triggered structural transition of an asymmetric $\mathrm{Cu}$ GB induced by $\mathrm{Ag}$ segregation observed by STEM and APT. Stable nanofacets are formed consisting of nanoscale asymmetric Ag-depleted $\{230\}$ / $\{100\}$ and Ag-rich symmetric $\{210\}$ segments. Combined MCMD simulations of a boundary with the same misorientation and asymmetric inclination reveal distinct faceting and facet stabilization by $\mathrm{Ag}$ segregation, supporting the experimental observations. Further simulations of different $\{210\}$ GB phases establish that the $\{210\}$ kite structure can accommodate the largest amount of $\mathrm{Ag}$ and should therefore be the most stable, as observed in the experiment. These types of segregation-induced faceting transitions with nanometer-sized facets can easily be overlooked, and only atomic resolution STEM is able to resolve them. The stabilization of nanometer-sized symmetric facets at an asymmetric GB indicates that these transitions can also occur for other GB inclinations whenever a solute has a low solubility in the bulk and symmetric GB structures with high solute adsorption exist.

Discussions with Blazej Grabowski and Baptiste Gault are acknowledged. The funding and support by the European Research Council (ERC) under the EU's Horizon 2020 Research and Innovation Programme is gratefully acknowledged by N.P. (Grant No. 639211) and G. D. (Grant No. 787446). Work at the Molecular Foundry was supported by the Office of Science, Office of Basic Energy Sciences of the U.S. Department of Energy under Contract No. DE-AC02-05CH11231 with support by the German Academic Exchange Service. The work at Lawrence Livermore National Laboratory (LLNL) was performed under the auspices of the U.S. Department of Energy by Lawrence Livermore National Laboratory under Contract No. DE-AC52-07NA27344. T. F. was funded by the Laboratory Directed Research and Development Program at LLNL under Project Tracking Code No. 17LW-012.
*To whom all correspondence should be addressed.

[1] A. Sutton and R. Balluffi, Interfaces in Crystalline Materials, Oxford Classic Texts in the Physical Sciences Vol. 51 (Oxford University, Oxford, 2006).

[2] P. R. Cantwell, M. Tang, S. J. Dillon, J. Luo, G. S. Rohrer, and M. P. Harmer, Acta Mater. 62, 1 (2014).

[3] R. Schweinfest, A. T. Paxton, and M. W. Finnis, Nature (London) 432, 1008 (2004).

[4] G. Duscher, M. F. Chisholm, U. Alber, and M. Rühle, Nat. Mater. 3, 621 (2004).

[5] S. V. Divinski, H. Edelhoff, and S. Prokofjev, Phys. Rev. B 85, 144104 (2012).

[6] S. J. Dillon, M. Tang, W. C. Carter, and M. P. Harmer, Acta Mater. 55, 6208 (2007).

[7] J. Luo, H. Cheng, K. M. Asl, C. J. Kiely, and M. P. Harmer, Science 333, 1730 (2011).

[8] J. Luo, H. Wang, and Y.-M. Chiang, J. Am. Ceram. Soc. 82, 916 (1999).

[9] J. W. Gibbs, The Collected Works of J. Willard Gibbs, Volume 1: Thermodynamics (Yale University Press, New Haven, CT, 1945).

[10] E. W. Hart, Scr. Metall. 2, 179 (1968).

[11] M. Tang, W. C. Carter, and R. M. Cannon, Phys. Rev. B 73, 024102 (2006).

[12] T. Frolov, D. L. Olmsted, M. Asta, and Y. Mishin, Nat. Commun. 4, 1899 (2013a).

[13] J. Rickman and J. Luo, Curr. Opin. Solid State Mater. Sci. 20, 225 (2016).

[14] J. Cahn, J. Phys. (Paris), Colloq. 43, C6-199 (1982).

[15] J. S. Choi and D. Y. Yoon, ISIJ International (1989-) (Iron and Steel Institute of Japan) 41, 478 (2001).

[16] A. Barg, E. Rabkin, and W. Gust, Acta Metall. Mater. 43, 4067 (1995).

[17] M. L. Bowers, C. Ophus, A. Gautam, F. Lancon, and U. Dahmen, Phys. Rev. Lett. 116, 106102 (2016).

[18] M. Tschopp and D. McDowell, Philos. Mag. 87, 3871 (2007).

[19] J. A. Brown and Y. Mishin, Phys. Rev. B 76, 134118 (2007).

[20] Z. Wu, Y. Zhang, and D. Srolovitz, Acta Mater. 57, 4278 (2009)

[21] J. C. Hamilton, D. J. Siegel, I. Daruka, and F. Léonard, Phys. Rev. Lett. 90, 246102 (2003).

[22] R. Hadian, B. Grabowski, M. W. Finnis, and J. Neugebauer, Phys. Rev. Mater. 2, 043601 (2018).

[23] W. Sigle, L.-S. Ciiang, and W. Gusr, Philos. Mag. A 82, 1595 (2002).

[24] T. Ference and R. Balluffi, Scr. Metall. 22, 1929 (1988).

[25] A. Kundu, K. M. Asl, J. Luo, and M. P. Harmer, Scr. Mater. 68, 146 (2013).

[26] A. Stoffers, B. Ziebarth, J. Barthel, O. Cojocaru-Mirédin, C. Elsässer, and D. Raabe, Phys. Rev. Lett. 115, 235502 (2015).

[27] D. Medlin, K. Hattar, J. Zimmerman, F. Abdeljawad, and S. Foiles, Acta Mater. 124, 383 (2017).

[28] T. Frolov, S. V. Divinski, M. Asta, and Y. Mishin, Phys. Rev. Lett. 110, 255502 (2013).

[29] See Supplemental Material at http://link.aps.org/ supplemental/10.1103/PhysRevLett.121.255502, which includes Refs. [12,30-34], for all of the experimental and simulation details. 
[30] K. Thompson, D. Lawrence, D. Larson, J. Olson, T. Kelly, and B. Gorman, Ultramicroscopy 107, 131 (2007).

[31] M. Miller, K. Russell, and G. Thompson, Ultramicroscopy 102, 287 (2005).

[32] P. Williams, Y. Mishin, and J. Hamilton, Model. Simul. Mater. Sci. Eng. 14, 817 (2006).

[33] C. Ophus, Adv. Struct. Chem. Imaging 3, 13 (2017).

[34] A. Pryor, Jr., C. Ophus, and J. Miao, Adv. Struct. Chem. Imaging 3, 15 (2017).

[35] J. R. McBride, T. C. Kippeny, S. J. Pennycook, and S. J. Rosenthal, Nano Lett. 4, 1279 (2004).

[36] N. J. Peter, C. H. Liebscher, C. Kirchlechner, and G. Dehm, J. Mater. Res. 32, 968 (2017).

[37] R. Hadian, B. Grabowski, C. P. Race, and J. Neugebauer, Phys. Rev. B 94, 165413 (2016).

[38] C. O'Brien, C. Barr, P. Price, K. Hattar, and S. Foiles, J. Mater. Sci. 53, 2911 (2018).
[39] P. J. Felfer, C. R. Killmore, J. G. Williams, K. R. Carpenter, S. P. Ringer, and J. M. Cairney, Acta Mater. 60, 5049 (2012).

[40] B. W. Krakauer and D. N. Seidman, Phys. Rev. B 48, 6724 (1993).

[41] S. Plimpton, J. Comput. Phys. 117, 1 (1995).

[42] T. Frolov, M. Asta, and Y. Mishin, Phys. Rev. B 92, 020103 (2015).

[43] B. Sadigh, P. Erhart, A. Stukowski, A. Caro, E. Martinez, and L. Zepeda-Ruiz, Phys. Rev. B 85, 184203 (2012).

[44] S. Yang, N. Zhou, H. Zheng, S. P. Ong, and J. Luo, Phys. Rev. Lett. 120, 085702 (2018).

[45] P. Subramanian and J. Perepezko, J. Phase Equilib. 14, 62 (1993).

[46] T. Frolov and Y. Mishin, Phys. Rev. B 85, 224107 (2012).

[47] G. Schusteritsch, T. D. Kühne, Z. X. Guo, and E. Kaxiras, Philos. Mag. 96, 2868 (2016).

[48] V. Razumovskiy, S. Divinski, and L. Romaner, Acta Mater. 147, 122 (2018). 